\title{
Beyond the Gleason score: the prognostic significance of prostate cancer subtypes
}

\author{
Trevor A. Flood ${ }^{1}$, Nicola Schieda ${ }^{2}$ \\ ${ }^{1}$ Department of Pathology and Laboratory Medicine, ${ }^{2}$ Department of Medical Imaging, The Ottawa Hospital, Ottawa, Canada \\ Correspondence to: Trevor A. Flood. Division of Anatomical Pathology, The Ottawa Hospital, University of Ottawa, 501 Smyth Road, 4th Floor CCW, \\ Room 4259, Ottawa, Ontario K1H 8L6, Canada. Email: tflood@toh.on.ca. \\ Response to: Hassan O, Matoso A. Clinical significance of subtypes of Gleason pattern 4 prostate cancer. Transl Androl Urol 2018. [Epub ahead of \\ print].
}

Submitted Mar 21, 2018. Accepted for publication Mar 26, 2018.

doi: $10.21037 /$ tau.2018.04.01

View this article at: http://dx.doi.org/10.21037/tau.2018.04.01

We read with great interest the excellent review by Drs. Hassan and Matoso regarding the "Clinical significance of subtypes of Gleason pattern 4 prostate cancer" (1). Our group has published several manuscripts on this important topic that were unfortunately not captured by the author's search of the current literature. We would therefore like to contribute our experience concerning this emerging subject to update your readership regarding its importance in clinical practice.

Our group has focused our investigations primarily on the significance of subtypes of Gleason pattern 4 identified in Gleason score $3+4=7$ tumors. This cohort of patients is relevant because occasionally men with small amounts of Gleason pattern 4 carcinoma identified on biopsy are considered for candidacy in active surveillance (AS) protocols. Our research has attempted to identify those patients who could benefit from this management strategy and also to identify patients who would potentially fail AS. In our first paper (2), we assessed the prognostic significance of detecting either small cribriform ( $\leq 12$ lumens) or large cribriform ( $>12$ lumens) subtypes on transrectal ultrasound (TRUS) guided biopsies in men with Gleason score $3+4=7$ carcinoma. Our results showed that the presence of cribriform morphology (regardless of size) on TRUS guided biopsy was significantly associated with stage $\geq$ pT3 on radical prostatectomy (RP). Other clinicopathologic features including patient age, serum prostate-specific antigen (PSA), PSA density, and nuclear/nucleolar prominence were not associated with $\geq \mathrm{pT} 3$ disease.

The results from this study prompted us to conduct a subsequent analysis in a larger cohort of patients where we examined all subtypes of Gleason pattern 4 detected on TRUS guided biopsy in patients with Gleason score 3+4=7 carcinoma (3). Clinicopathologic parameters including age, PSA, PSA density, and percentage core involved by Gleason pattern 4 were not correlated with stage $\geq \mathrm{pT} 3$ or grade $>3+4=7$ at RP. TRUS guided biopsies were assessed for the presence/absence of all Gleason pattern 4 subtypes (poorly/ill-defined glands, fused glands, glomerulations, and cribriform morphology). The inter-observer agreement was strong for both small and large cribriform morphology and variably moderate to strong for poorly/ill-defined glands, fused glands, and glomerulations. Our degree of agreement for the non-cribriform subtypes was higher than that reported in the literature (4) and we postulated that identifying morphologies on biopsy may be less challenging than identifying subtypes on larger fragments of tissue that are seen with RP specimens. Our analysis again revealed that only cribriform morphology (of any size) identified on TRUS guided biopsy was associated with stage $\geq \mathrm{pT} 3$ and grade $>3+4=7$ on RP. Other Gleason pattern 4 subtypes were not associated with adverse features on RP. We also found that an increasing number of subtypes detected on biopsy were associated with an increased likelihood of adverse features at RP. We, as others have, postulated that this is likely because high grade carcinoma is typically associated with increased architectural heterogeneity (4). Our group's findings are concordant with what has been reported in the literature; namely that the cribriform morphology (regardless of size) represents a more aggressive subtype of 
Gleason pattern 4 tumor. Our data indicate that cribriform architecture detected on TRUS biopsy in patients with Gleason score $3+4=7$ prostate cancer should be considered a contraindication to AS and we agree with Drs. Hassan and Matoso that the presence of this subtype should be reported.

In our most recent paper we expanded the horizon of the prognostic value of prostate cancer subtypes and examined the significance of cancer morphologies in Gleason pattern 5 tumors (5). A diagnosis of Gleason pattern 5 is reserved for malignant cells that do not show signs of glandular differentiation and includes: (I) single cells; (II) sheets of cells; (III) cords; and (IV) comedonecrosis within solid tumor nests or within invasive papillary/ cribriform structures. Two new morphologies that were recently added to the pattern 5 group are solid medium to large nests with rosette-like spaces, and small solid cylinders (6). We evaluated for the presence of Gleason pattern 5 morphologies in RPs and determined which subtypes were associated with biochemical recurrence (BCR) in patients who were treated with RP as monotherapy. We found that, much like Gleason pattern 4 carcinomas; Gleason pattern 5 lesions may also represent a biologically heterogeneous group. Certain pattern 5 subtypes were significantly associated with BCR. These subtypes included malignant cells with comedonecrosis, sheets of malignant cells, and small solid cylinders. Similar to our findings regarding Gleason pattern 4 subtypes, poorly differentiated Gleason pattern 5 tumors with an increased number of subtypes were strongly associated with BCR. We concluded that an increased morphologic heterogeneity is associated with aggressive tumor behavior. Although it is considered a member of the Gleason pattern 4 group, we included cribriform morphology in our analysis and although this subtype was observed in conjunction with $98.7 \%$ of Gleason pattern 5 tumors, it was not in itself associated with BCR.

The Gleason scoring system remains an extremely important predictor of tumor aggressiveness but it is becoming increasingly evident that additional prognostic information can be obtained by assessing for prostate cancer morphologic subtypes. We appreciate the work performed

Cite this article as: Flood TA, Schieda N. Beyond the Gleason score: the prognostic significance of prostate cancer subtypes. Transl Androl Urol 2018;7(Suppl 2):S260-S261. doi: 10.21037/ tau.2018.04.01 by Drs. Hassan and Matoso in synthesizing their review on this interesting subject matter and we look forward to exciting new developments regarding this important topic.

\section{Acknowledgements}

None.

\section{Footnote}

Conflicts of Interest: The authors have no conflicts of interest to declare.

\section{References}

1. Hassan O, Matoso A. Clinical significance of subtypes of Gleason pattern 4 prostate cancer. Transl Androl Urol 2018. [Epub ahead of print].

2. Keefe DT, Schieda N, El Hallani S, et al. Cribriform morphology predicts upstaging after radical prostatectomy in patients with Gleason score $3+4=7$ prostate cancer at transrectal ultrasound (TRUS)-guided needle biopsy. Virchows Arch 2015;467:437-42.

3. Flood TA, Schieda N, Keefe DT, et al. Utility of Gleason pattern 4 morphologies detected on transrectal ultrasound (TRUS)-guided biopsies for prediction of upgrading or upstaging in Gleason score 3+4=7 prostate cancer. Virchows Arch 2016;469:313-9.

4. Dong F, Yang P, Wang C, et al. Architectural heterogeneity and cribriform pattern predict adverse clinical outcome for Gleason grade 4 prostatic adenocarcinoma. Am J Surg Pathol 2013;37;1855-61.

5. Flood TA, Schieda N, Sim J, et al. Evaluation of tumor morphologies and association with biochemical recurrence after radical prostatectomy in grade group 5 prostate cancer. Virchows Arch 2018;472:205-12.

6. Epstein JI, Egevad L, Amin MB, et al. The 2014 International Society of Urological Pathology (ISUP) Consensus Conference on Gleason Grading of Prostatic Carcinoma: Definition of Grading Patterns and Proposal for a New Grading System. Am J Surg Pathol 2016;40:244-52. 